\title{
Pathogénie de la maladie de Berger \\ Implication des immunoglobulines A et de leurs récepteurs
}

Renato C. Monteiro, Valérie Leroy, Pierre Launay, Ivan Cruz Moura, Michelle Arcos-Fajardo, Marc Benhamou, Élie Haddad

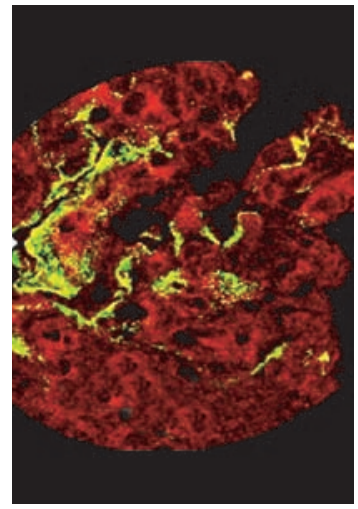

rénales en France concernent des patients atteints de néphropathie à $\operatorname{lgA}[2]$. La néphropathie à $\lg A$ est définie aujourd'hui encore par les critères immunohistologiques décrits en 1968 par J. Berger et N. Hinglais [3]. Elle est caractérisée par des dépôts mésangiaux d'IgA, exclusivement de la sous-classe IgAl, et par une prolifération mésangiale (Figure 1). Au sein des dépôts mésangiaux sont également présents la fraction C 3 du complément ( $70 \%$ des cas), des IgG ( $40 \%$ des cas) et, plus rarement (5 à $10 \%$ des cas), des IgM [3, 4]. En microscopie optique, les aspects lésionnels sont variables, allant d'une simple hypertrophie de la matrice mésangiale à une augmentation importante de la cellularité mésangiale. Les lésions sont segmentaires (une partie du floculus est atteinte au sein du glomérule) et focales (une partie seulement des glomérules est concernée) ou diffuses. Elles s'associent parfois à une nécrose du floculus et à une prolifération extracapillaire des cellules épithéliales formant des croissants épithéliaux. Ces lésions peuvent évoluer vers une sclérose glomérulaire irréversible. En microscopie électronique, on observe des 
dépôts denses dans les espaces intercellulaires mésangiaux et le long de la membrane basale glomérulaire.

Deux tableaux cliniques sont habituels: I'association asymptomatique d'une hématurie microscopique et d'une protéinurie ou des épisodes récidivants d'hématurie macroscopique. Ces hématuries macroscopiques apparaissent souvent 24 à 48 heures après un épisode infectieux des voies aériennes supérieures ou, plus rarement, au cours d'un effort physique. L'absence d'atteinte extrarénale permet de distinguer la maladie de Berger de la glomérulonéphrite du purpura rhumatoïde, également caractérisée par des dépôts mésangiaux d'IgAl, mais comportant des signes de vascularite cutanée, digestive et articulaire. Les tableaux cliniques de la néphropathie à IgA et du purpura rhumatoïde sont classiquement très différents. Cependant, certains symptômes tels que les arthralgies ou les douleurs abdominales, fréquents au cours du purpura rhumatoïde, peuvent être observés au cours d'une maladie de Berger et quelques enfants présentent, plusieurs années après une poussée de purpura rhumatoïde, des épisodes isolés d'hématurie macroscopique. Enfin, des formes familiales ont été décrites où coexistaient, au sein d'une même famille, des cas de néphropathie à IgA et de purpura rhumatoïde, soulignant les rapports étroits entre ces deux affections [5].

Les mécanismes physiopathogéniques de la maladie de Berger restent en grande partie inconnus. Leur compréhension, essentielle pour mettre au point des stratégies thérapeutiques innovantes et performantes, a connu des progrès récents. Les recherches se sont orientées essentiellement vers l'étude de l'IgAl, qui constitue la sousclasse d'IgA exclusivement déposée dans le mésangium, et des différents récepteurs pour les IgA.

\section{Anomalie de la réponse immune IgA}

\section{Rôle pathogène des complexes immuns} contenant des IgA

L'existence d'un facteur sérique à l'origine de la maladie est étayée par de nombreuses observations cliniques. Après transplantation rénale chez un patient atteint de néphropathie à $\lg A$, les dépôts mésangiaux d'IgA se reconstituent dans le rein greffé dans environ un tiers des cas $[6,7]$. Inversement, les dépôts d'IgA d'un rein atteint de néphropathie à IgA disparaissent lorsqu'il est greffé à un individu souffrant d'une autre affection rénale [8].

L'analyse du sérum et des glomérules des patients a suggéré le rôle pathogène des IgA elles-mêmes et notamment des complexes immuns contenant des IgA. En effet, la concentration d'IgA sériques est augmentée chez plus de $50 \%$ des patients. Cette augmentation porte à la fois sur les formes poly- et monomériques mais le rapport polymériques/monomériques est très augmenté. Par ailleurs, des complexes immuns à $\operatorname{lgA}$, absents chez les individus sains, ont été isolés à la fois dans le sang [9] et dans les glomérules des patients [10]. Les IgAl polymériques sont les principaux composants aussi bien des complexes immuns circulants que de ceux élués des glomérules suggérant que ces derniers ont bien une origine sérique $[9,10]$. Le rôle des complexes immuns circulants dans le développement de la maladie est appuyé par certains modèles animaux de néphropathie à IgA où une glomérulonéphrite est induite par l'injection intraveineuse de complexes immuns à IgA préformés de grande taille [11]. Toutefois, ces observations ne présagent pas de la nature de complexes antigène-anticorps des complexes immuns à IgA isolés chez les patients. De fait, aucun antigène spécifique de la maladie et commun à tous les patients n'a été isolé au sein de ces complexes à IgA et les IgA éluées des biopsies rénales de patients atteints de néphropathie à IgA n'ont pas de spécificité particulière [12]. Le rôle, dans la formation des complexes immuns, d'une réponse immune excessive à un antigène paraît donc peu probable. Une anomalie intrinsèque du système immunitaire conduisant à une augmentation globale de la synthèse des IgA, à l'origine de la formation des complexes immuns, est une des hypothèses proposées.

\section{Anomalie de la régulation de la production des IgA}

Le site de la production accrue des IgA - médullaire ou muqueux - chez les patients atteints de néphropathie à IgA est encore débattu. Chez les individus nor-

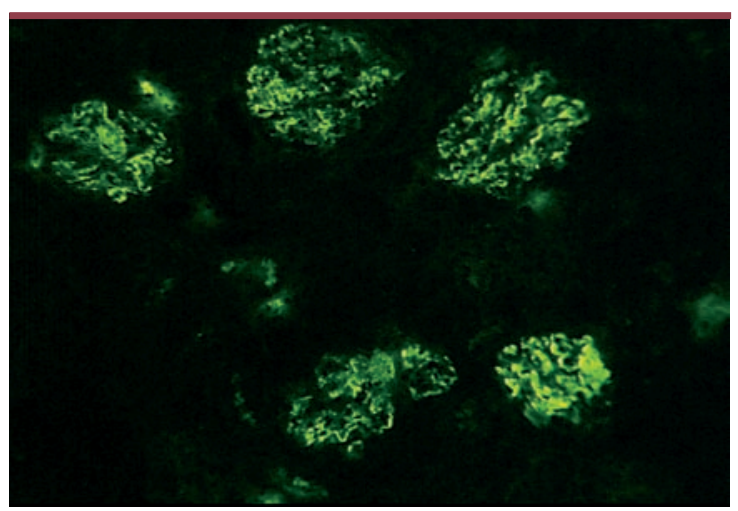

Figure 1. Dépôts mésangiaux d'IgA. Les dépôts d'IgA sont révélés par immunofluorescence directe à l'aide d'anticorps fluorescents anti-IgA humaines au cours de la maladie de Berger. Les dépôts d'IgA sont exclusivement localisés dans le glomérule et l'axe mésangial (cliché Pr Michel Peuchmaur, Hôpital Robert Debré). 
maux, les IgA sériques sont de façon prédominante de la sous classe des IgAl, monomériques et produites par les plasmocytes de la moelle osseuse tandis que les IgA sécrétoires, synthétisées principalement par les plasmocytes du chorion sont de classe IgAl et IgA2 et polymériques. Les principaux arguments en faveur d'une anomalie de la réponse immune affectant le compartiment médullaire sont l'absence d'IgA2 dans les dépôts mésangiaux et l'augmentation des concentrations sériques d'IgA concernant exclusivement la sous classe des IgAl. Des études cliniques ont montré une diminution de la réponse IgA sécrétoires au sein des muqueuses lors d'une immunisation par voie muqueuse tandis que la production de IgA polymériques sériques en réponse à une infection chronique muqueuse ou une immunisation systémique était exagérée [13-15]. Un déficit de la réponse immune IgA dans les muqueuses pourrait ainsi être responsable d'un passage anormal d'antigènes dans la circulation et d'une stimulation antigénique récurrente ayant pour conséquence une production accrue d'IgA dans la moelle osseuse [13].

Néanmoins, quel que soit le mécanisme d'élévation des concentrations sériques d'lgA, l'augmentation de la quantité d'IgA ne suffit pas à expliquer la formation des complexes immuns à IgA et les dépôts dans le mésangium. En effet, les patients atteints de Sida ou de myélome à IgA ont des concentrations d'lgA sériques très élevées et présentent cependant rarement une néphropathie à $\lg A$.

\section{Anomalies de structure des IgAl}

La constatation que les IgAl mésangiales présentaient une charge négative a conduit à rechercher d'éventuelles anomalies de structure de ces molécules [10]. Les IgAl présentent une particularité structurale

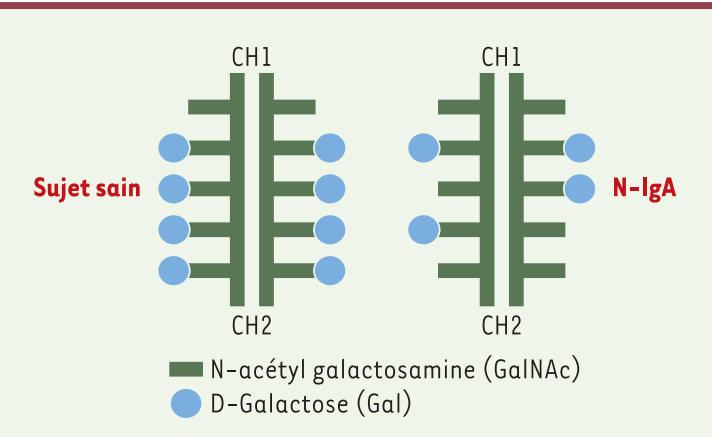

Figure 2. Anomalies des 0 -glycosides de la région charnière des IgAl chez les patients présentant une néphropathie à IgA ( $N$ $\lg A$ ) (d'après [16]). $\mathrm{CH} 1$ : constant heavy chain $1, \mathrm{CH} 2$ : constant heavy chain 2. remarquable: contrairement aux IgA2, elles possèdent dans leur région charnière des résidus $\mathrm{N}$-acétyl galactosamine- $\beta$ 1,3 galactose 0 -glycosylés. II a été montré par différentes techniques, notamment l'étude de l'affinité des IgA pour différentes lectines et l'analyse directe de leur composition en sucres, qu'une fraction des molécules d'IgAl sériques des patients atteints de néphropathie à IgA avaient un défaut de galactosylation de ces résidus (Figure 2) [16]. Les IgAl isolées des dépôts glomérulaires de patients avec néphropathie à IgA présentent les mêmes anomalies structurales [17]. Deux observations suggèrent que l'hypogalactosylation des IgAl joue un rôle central dans la genèse des dépôts rénaux d'IgA. D'une part, la présence d'IgAl hypogalactosylées a été mise en évidence dans le purpura rhumatoïde avec atteinte rénale alors que, dans les formes sans atteinte rénale, les IgAl sont de structure normale [18]. D'autre part, des IgA humaines injectées chez le rat ne s'accumulent dans les glomérules que lorsqu'elles ont été préalablement dégalactosylées in vitro [19]. L'origine du déficit de galactosylation des IgAl au cours de la maladie de Berger ou de la néphropathie du purpura rhumatoïde n'est pas encore élucidée. L'hypothèse d'une modification de la séquence des acides aminés de la région charnière a été réfutée $[20,21]$. Un déficit en $\beta 1-3$ galactosyltransférase pourrait rendre compte de cette hypogalactosylation des IgAl. Une étude a, en effet, rapporté une diminution de l'activité de cet enzyme dans les cellules B périphériques des patients atteints de néphropathie à IgA [22]. Cette observation demande cependant à être confirmée, notamment dans les plasmocytes médullaires, producteurs d'IgA.

\section{Quelles sont les conséquences biologiques de l'hypogalactosylation?}

De nombreuses hypothèses ont été proposées pour expliquer comment ces IgAl anormales participent à la physiopathologie de la néphropathie à $\lg A$. L'altération des sucres pourrait, par différents mécanismes, favoriser la formation de complexes immuns à $\lg A$ et leurs dépôts dans le mésangium. T. Kokubo et al. ont ainsi montré qu'in vitro, les IgAl hypogalactosylées formaient spontanément des agrégats et que leur affinité pour différents composants de la matrice mésangiale (laminine, fibronectine, collagène IV) était augmentée [23]. D'après les travaux de J. Mestecky et al., les IgA hypogalactosylées sont présentes dans le sérum sous forme de complexes contenant à la fois des IgA et des IgG. Les $\mathrm{N}$-acétyl galactosamines terminales exposées constitueraient des néo-épitopes reconnus par des IgA et des IgG anti-IgA naturels conduisant à la formation des complexes immuns à IgA $[24,25]$. Ces complexes 
échapperaient au catabolisme hépatique. En effet, les hépatocytes et, à un moindre degré, les cellules hépatiques non parenchymateuses, participent normalement au catabolisme des IgA via le récepteur des asialoglycoprotéines. Au sein des complexes immuns à IgA, les résidus sialiques de la région charnière normalement reconnus par le récepteur des asialoglycoprotéines seraient masqués, empêchant ainsi la fixation des complexes au récepteur et leur clairance. Une autre hypothèse, développée par notre équipe, est que l'hypogalactosylation des IgAl est responsable d'une interaction anormale des IgAl avec leurs différents récepteurs exprimés par les cellules mésangiales ou d'autres types cellulaires.

\section{l'apport de modèles murins}

\section{Interaction anormale des IgA avec le CD89:}

\begin{abstract}
Anomalie du CD89 chez les patients atteints de néphropathie à IgA une plus forte affinité pour les IgA polymériques que pour les monomériques. Il participe à la fois aux défenses immunitaires et au catabolisme des IgA [26]. Chez les patients atteints de néphropathie à IgA, l'expression membranaire du CD89 à la surface des monocytes est diminuée malgré une synthèse intracellulaire normale de la protéine [27]. Par ailleurs, nous avons mis en évidence dans le sang des patients la présence d'une forme soluble de CD89, absente chez les individus sains, liée à des IgA sous forme de complexes circulants CD89-IgA [28].
\end{abstract} Le premier récepteur des IgA identifié, le CD89 ou RFc $\alpha$, est une glycoprotéine transmembranaire de 266 acides aminés ( 55 à $75 \mathrm{kDa}$ ), exprimée constitutivement chez l'homme par les cellules de la lignée monocytaire (Figure 3). Le CD89 fixe les IgAl et IgA2, avec

\section{Nouveau modèle murin de néphropathie à IgA: les souris transgéniques pour le CD89 humain} Les modèles expérimentaux initialement décrits se différenciaient de la pathologie humaine par différents aspects: la souris ddy présente une augmentation de la concentration sérique d'IgA mais pas d'hématurie [29] alors que la souris dont le gène codant pour l'utéroglobine* a été invalidé, développe des dépôts rénaux constitués certes d'IgA, mais surtout de fibronectine [30].

Nous avons développé des souris transgéniques pour le CD89 humain dont le transgène est sous contrôle d'un promoteur spécifique de la lignée monocytaire-macro-

\footnotetext{
* Molécule sérique qui se lie à la fibronectine et règle son taux sérique.
}

phagique. Ces souris transgéniques pour le CD89 humain développent spontanément une pathologie similaire à la néphropathie à IgA: dépôts mésangiaux massifs d'IgA, infiltration glomérulaire et interstitielle par des macrophages, expansion de la matrice mésangiale, hématurie et protéinurie modérée [28]. Dans le sérum de ces souris transgéniques, une forme soluble du CD89 et des complexes CD89 soluble-IgA sont détectés. L'injection du sérum de ces souris à des souris non transgéniques transmet la maladie, alors que le même sérum, déplété du CD89 soluble, n'induit aucune pathologie (Figure 4) démontrant le rôle clé du CD89 soluble dans le développement de la maladie.

Le rôle des IgA dans l'apparition de la forme soluble du CD89 a été démontré chez des souris SCID (qui présentent un déficit immunitaire humoral complet et n'ont donc aucune IgA circulante) chez lesquelles le gène du CD89 humain a été exprimé. Ces souris ne développent pas spontanément la maladie; le CD89 soluble n'est pas détectable dans leur sérum, expliquant l'absence de pathologie rénale, alors que l'expression du CD89 à la surface des cellules sanguines est jusqu'à dix fois supérieure à l'expression observée chez les souris transgéniques immunocompétentes.

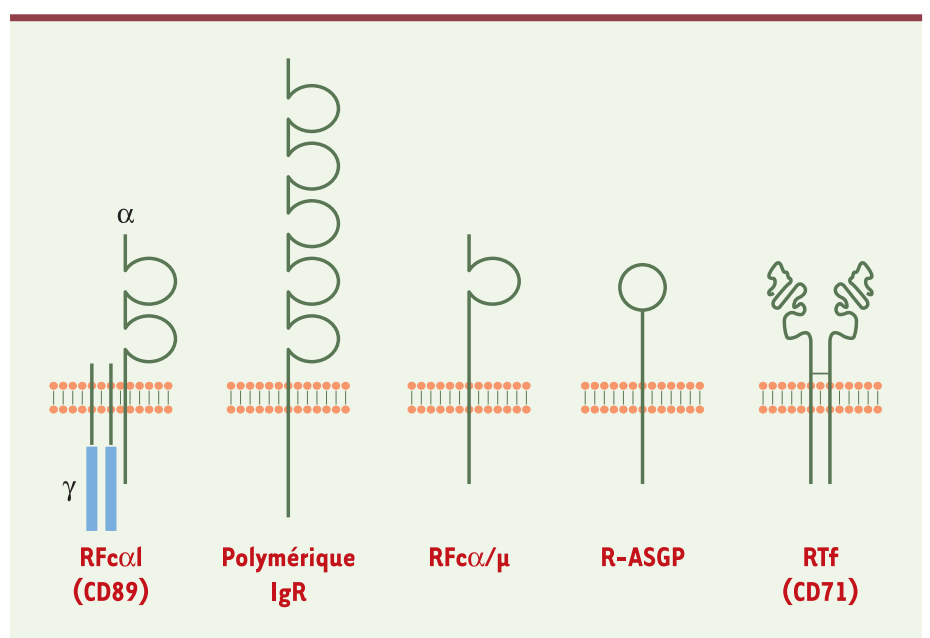

Figure 3. Les différents récepteurs des IgA. Le RFcal (CD89) (récepteur monospécifique pour les IgA humaines), exprimé par les cellules de la lignée monocytaire-macrophagique et les polynucléaires neutrophiles, participe aux défenses immunitaires (phagocytose des particules opsonisées [recouvertes d'anticorps], présentation de l'antigène aux cellules compétentes...) et au catabolisme des IgA. Le récepteur des immunoglobulines polymériques, présent sur les cellules épithéliales des muqueuses et des glandes exocrines, permet la transcytose des IgA sécrétoires vers la lumière des organes. Le récepteur des asialoglycoprotéines (R-ASGP) présent sur les cellules hépatiques contribue à la clairance des IgA notamment les IgA2. Les caractéristiques fonctionnelles du $\mathrm{RF} c \alpha / \mu$ (récepteur multispécifique que lient à la fois les $\lg A$ et les $\lg M$ ) et du récepteur de la transferrine (RTf) sont encore inconnues. 
Chez ces souris, une néphropathie à IgA peut être induite par l'injection d'IgA isolées à partir du sang des patients atteints de néphropathie à IgA, les IgA de sujets sains n'induisant pas la maladie (Figure 4). L'injection des IgA de patients est suivie d'une diminution de l'expression membranaire du CD89 sur les cellules sanguines circulantes puis de l'apparition de dépôts mésangiaux d'IgA [28].

L'ensemble de ces résultats démontre que, dans ce modèle, à la fois le CD89 et les IgA anormales sont nécessaires au développement d'une néphropathie à $\lg$. Néanmoins, la survenue d'une néphropathie à IgA chez des souris transgéniques pour le CD89 et sans déficit immunitaire (c'est-à-dire en présence des IgA normales de la souris) est inattendue. Une explication pourrait venir de la forme des IgA sériques murines qui, contrairement à ce qui est observé chez l'homme sain, sont de façon constitutive essentiellement polymériques.

Rôle du CD89 dans le développement de la néphropathie à IgA: modèle physiopathologique

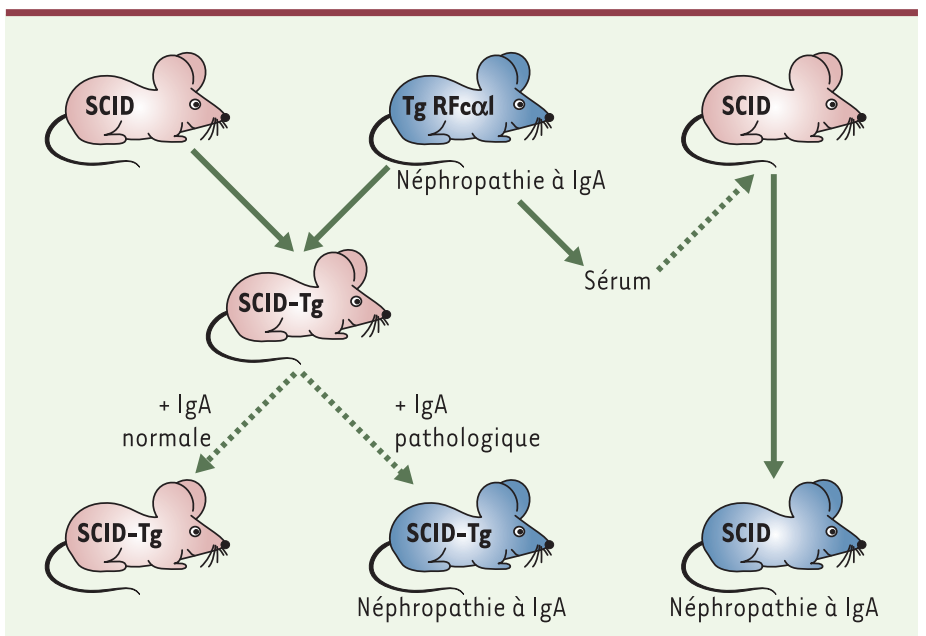

Figure 4. Modèle des souris transgéniques pour le récepteur Fcal (RFcal ou CD89) humain. Les souris transgéniques (Tg RFcal) présentent une pathologie similaire à la néphropathie à IgA (en bleu). Les souris immunodéficientes SCID (en rose), qui ne possèdent pas d'IgA sériques, ont été croisées avec les souris transgéniques. Les souris SCID transgéniques (SCID-Tg) ne développent pas spontanément la maladie (en rose). L'injection d'IgA de patients provoque l'apparition de la néphropathie à IgA alors que l'injection d'IgA d'individus normaux ne déclenchent pas la maladie. Les IgA anormales constituent un facteur pathogène indispensable au développement d'une néphropathie à IgA. Le rôle du RFcal a été démontré par expériences de transfert passif. Le sérum de souris Tg RFcal induit la maladie chez les souris SCID alors que le même sérum déplété du CD89 soluble ne provoque plus la maladie. Le CD89 soluble complexé aux IgA a donc un rôle primordial dans la genèse de la néphropathie à IgA.
Les anomalies du CD89 observées chez les patients atteints de néphropathie à IgA et l'étude des souris transgéniques nous conduisent à proposer le mécanisme physiopathogénique suivant: la fixation des IgA anormales sur le CD89 entraînerait une libération de la partie extracellulaire du récepteur et une diminution $d u$ CD89 membranaire. La partie extracellulaire du CD89 boucle d'auto-aggravation se mettrait alors en IgA polymériques et de leur interaction déposeraient alors dans les glomérules par liaison à des récepteurs de surface des cellules mésangiales ayant une affinité pour ces molécules (Figure 4).

Le mécanisme moléculaire précis du relargage du CD89 soluble est encore inconnu. L'hypothèse que nous formulons est l'existence d'une co-agrégation du CD89 avec une protéase membranaire. Cette hypothèse est confortée par une série d'expériences in vitro. $\varepsilon n$ effet, l'incubation de cellules sanguines isolées des patients en présence ou en l'absence de sérum autologue montre que les IgA sont nécessaires au maintien de la diminution de l'expression du récepteur à la surface des cellules [27]. Par ailleurs, dans un modèle cellules transfectées avec le CD89, la production de CD89 soluble est induite par des IgA polymériques [28]. Enfin, un anticorps spécifique de la région intracytoplasmique du CD 89 n'est pas réactif vis-à-vis de la forme soluble du CD89 isolée du sang des patients, confirmant que seule la région extracellulaire du CD89 est présente au sein des complexes CD89 soluble-IgA. Malgré la forte suspicion que les complexes sériques CD89 soluble-IgA sont bien la forme sous laquelle les IgA se déposent dans le mésangium. La présence de CD89 n'a jamais été mise directement en évidence dans le mésangium des souris transgéniques pour le CD89 ou dans celui des patients. Cela pourrait s'expliquer par un défaut de sensibilité des anticorps utilisés lors du marquage immunohistochimique ou encore par le masquage des épitopes reconnus par l'anticorps lors de la liaison des complexes CD89 soluble-IgA au mésangium.

Le rôle du CD89 n'est pas restreint à la seule néphropathie à IgA primitive mais a été montré également dans les néphropathie à IgA secondaires des cirrhoses alcooliques, du Sida ou de la spondylarthrite ankylosante, affections où l'on observe une augmentation des concentrations sériques d'IgA et la présence de com- 
plexes immuns à $\operatorname{IgA}$. Une diminution de l'expression membranaire du CD89 sur les monocytes sanguins a, en effet, été observée dans toutes ces pathologies [3133]. Toutefois, le CD89 dans sa forme soluble n'a pas été isolé au cours des néphropathies à IgA secondaires [28] et le type d'IgA dont les concentrations sériques sont augmentées varie sensiblement selon l'affection considérée. Ainsi, dans la spondylarthrite ankylosante, les IgA sériques sont essentiellement monomériques [33] alors que dans la cirrhose, les IgA des deux sousclasses (IgAl et IgA2) sont augmentées et le rapport monomères/polymères reste normal [31]. De plus, contrairement à la néphropathie à IgA primitive, aucune anomalie de glycosylation des IgA n'a été mise en évidence dans ces trois affections. Ces différences suggèrent que le CD89 pourrait participer au développement des dépôts mésangiaux d'IgA au cours des néphropathies à IgA primitives ou secondaires, soit indirectement par le biais d'un défaut de catabolisme des IgA, soit directement via la production de complexes CD89 soluble-IgA. Les anomalies de l'interaction CD89-IgA procèdent probablement de mécanismes variables impliquant diversement des altérations du récepteur lui-même et/ou des IgA.

\section{Mécanisme de fixation des IgA aux cellules mésangiales: expression accrue d'un nouveau récepteur des IgAl par les cellules mésangiales}

Les nouveaux récepteurs des IgA:

récepteur de la transferrine et $\mathrm{RFc} \alpha / \mu$

Les IgA se lient aux cellules mésangiales de manière dépendante de la dose et saturable [34]. Cette observation rend l'existence d'un récepteur mésangial des IgA très probable. Cependant, aucun des récepteurs connus des IgA (CD89, récepteur des asialoglycoprotéines, récepteur des immunoglobulines polymériques) n'a pu être mis en évidence sur les cellules mésangiales [35].

Le RFc $\alpha / \mu$ a été proposé comme candidat potentiel. Chez la souris, il fixe les IgA mono- et polymériques, mais aussi les IgM avec une plus forte affinité [36]. Chez l'homme, son ARN messager a été détecté dans les cellules mésangiales humaines en culture [37]. Cependant, il n'existe aucune étude confirmant l'expression membranaire de la protéine. De plus, la faible fréquence des dépôts d'IgM dans la néphropathie à IgA rend douteuse l'implication du RFc $\alpha / \mu$ dans la pathogénie de cette affection.

Expression accrue du RTf dans la néphropathie à IgA Récemment, un autre récepteur des IgA a été caracté- risé. Il s'agit d'une glycoprotéine homodimérique de $180 \mathrm{kD}$, présente sur les cellules mésangiales en culture, qui a été identifiée comme le récepteur de la transferrine (RTf ou CD71) [38]. Le CD71 lie les IgAl et non les $\lg A 2$, et davantage les IgA polymériques que les IgA monomériques.

Des études immunohistochimiques sur biopsies rénales révèlent une expression augmentée du RTf dans le mésangium des patients atteints de néphropathie à IgA ou de purpura rhumatoïde avec atteinte rénale alors que le RTf est quasiment indétectable par les mêmes techniques dans des glomérules de rein normal $[38,39]$. L'augmentation de l'expression mésangiale du RTf n'est toutefois pas spécifique de la néphropathie à IgA puisqu'elle a également été observée chez des patients présentant d'autres types de glomérulonéphrite. Toutefois, le RTf n'est surexprimé que dans les glomérulonéphrites s'accompagnant de dépôts mésangiaux d'IgA et l'étude des glomérules en microscopie confocale après double marquage des IgA et du RTf par des fluorochromes montre une colocalisation de ces deux molécules [39]. Cette observation suggère que le récepteur des IgA mésangial est bien le RTf. Le mécanisme de son hyperexpression dans les glomérulonéphrites avec dépôts d'IgA reste cependant à déterminer.

\section{Conséquences de la fixation des IgA au mésangium}

Alors que les mécanismes responsables des dépôts d'IgA dans le mésangium apparaissent assez spécifiques des néphropathies à $\lg A$, les événements qui conduisent à la progression de la maladie (infiltration rénale par des monocytes-macrophages, prolifération épithéliale, lésions inflammatoires et fibreuses des glomérules) semblent communs à toutes les maladies glomérulaires chroniques. La liaison des IgAl aux cellules mésangiales induit, in vitro, chez la souris comme chez l'homme, une prolifération mésangiale et la sécrétion par les cellules mésangiales de cytokines pro-inflammatoires (interleukine 6, interleukine 1, tumor necrosis factor $\alpha$, transforming growth factor $\beta$ ) et de chimiokines telles que l'interleukine 8, le macrophage-inflammatory protein (MIP) et l'interferon-inducible protein 10 (IP-10) [40-42]. Ces médiateurs, par une activité paracrine ou autocrine, pourraient être responsables de l'infiltration du mésangium par les cellules sanguines mononucléées et des lésions inflammatoires.

La nature des facteurs responsables de la grande variabilité clinique et histopathologique de la néphropathie à IgA avec une évolution vers la sclérose glomérulaire et l'insuffisance rénale terminale pour certains patients ou la guérison pour d'autres, n'est pas encore élucidée. Facteurs génétiques dans la néphropathie à IgA 
Bien que la néphropathie à IgA soit le plus souvent une affection sporadique, des formes familiales ont été rapportées, qui représenteraient, d'après certains, plus de $50 \%$ des cas de néphropathie à IgA [43]. L'existence de facteurs génétiques prédisposant à la néphropathie à IgA est donc probable, même si la maladie ne présente pas un schéma de transmission simple, de type mendélien. Parmi les hypothèses explorées, le rôle dans le déclenchement de la maladie des antigènes du système HLA, du polymorphisme du $T$ cell receptor, du promoteur du gène codant pour le CD89 ou pour les immunoglobulines A ou la responsabilité du polymorphisme de l'enzyme de conversion de l'angiotensine dans la progression de la néphropathie à IgA vers l'insuffisance rénale terminale ont fait l'objet de plusieurs études (pour revue, voir [44]). Malheureusement, les résultats, souvent contradictoires, n'ont pas permis d'identifier le ou les gènes de susceptibilité pour la néphropathie à IgA.

L'approche la plus prometteuse actuellement semble être celle de A. Ghavari et al. qui ont réalisé une analyse de liaison sur la totalité du génome dans 30 familles de néphropathie à IgA [45]. De façon surprenante, aucune liaison significative n'a pu être établie entre la néphropathie à IgA et les principaux gènes habituellement proposés comme candidats (gène des immunoglobulines, antigènes majeurs d'histocompatibilité, gènes codant pour le CD89, l'utéroglobuline ou la galactosyl transfé- rase). Toutefois, cette étude a montré une association étroite entre néphropathie à IgA et le locus 6p22-23 dans $60 \%$ des familles analysées. D'autres études sont maintenant nécessaires pour caractériser le gène correspondant à ce locus de susceptibilité et expliquer les variations phénotypiques entre individus porteurs du trait 6p22-23.

\section{Conclusions}

L'ensemble des données sur les IgA et leurs récepteurs conduisent à proposer un nouveau mécanisme physiopathogénique dans la maladie de Berger (Figure 5). La fixation des IgAl de patients, anormalement glycosylées, sur le CD89 entraînerait un clivage de sa partie extracellulaire et une diminution de son expression membranaire. Une boucle d'auto-aggravation se mettrait alors en place résultant dans la diminution de la clairance des IgA polymériques et une augmentation des IgA polymériques complexées au CD89 soluble. Ces complexes se déposeraient alors dans les glomérules par liaison au récepteur de la transferrine surexprimé par les cellules mésangiales. Le développement de molécules inhibant la fixation des IgA à leurs récepteurs pourrait ainsi ouvrir une nouvelle voie dans la thérapie de la maladie de Berger. $\diamond$

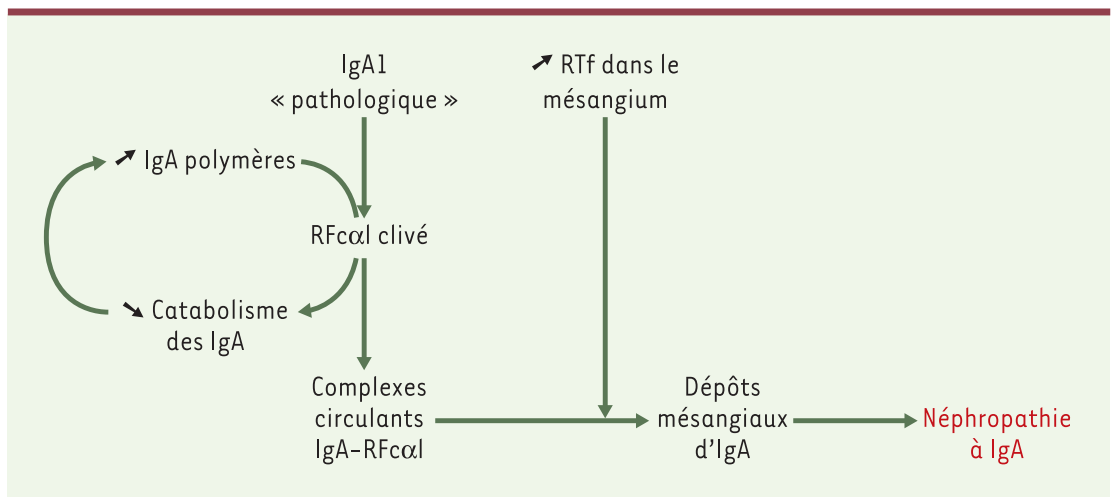

Figure 5. Schéma hypothétique du rôle pathogène du récepteur Fcol (RFcol ou CD89) et du récepteur de la transferrine (RTf) dans la néphropathie à IgA. La fixation des IgA hypogalactosylées sur le RFcal provoque le clivage et la libération de la partie extracellulaire du récepteur. Ce phénomène a deux conséquences: (1) la diminution de l'expression membranaire du RFcal sur les monocytes circulants avec, pour conséquence, une diminution de la clairance des IgA polymères; (2) le RFcal soluble libéré lie les IgA polymères sériques formant des complexes circulants IgA-RFcal. Ces complexes se déposent dans le rein par liaison au RTf, dont l'expression à la surface des cellules mésangiales est augmentée, conduisant au développement de la néphropathie à $\lg$.

\section{RÉFÉRENCES}

1. Galla JH. IgA nephropathy. Kidney Int 1995; 47: 377-87.

2. Simon P, Ramee MP, Autuly $V$, et al. Epidemiology of primary glomerular diseases in a French region. Variations according to period andage. Kidney Int 1994; 46: 1192-8.

3. Berger J, Hinglais N. Les dépôts intercapillaires d'IgA-IgG. J Urol Néphrol 1968; 74: 694-5.

4. Aucouturier P, Monteiro RC, Noel LH, Preud'homme JL, Lesavre P. Glomerular and serum immunoglobulin $G$ subclasses in IgA nephropathy. Clin Immunol Immunopathol 1989; 51: 338-47. 


\section{SUMMARY}

Pathogenesis of Berger's disease:

Recent advances on the involvement

of immunoglobulin $A$ and their receptors

Immunoglobulin A ( $\operatorname{IgA}$ ) nephropathy or Berger's disease is the most common form of primary glomerulonephritis in the world and one of the first cause of end-stage renal failure. IgA nephropathy is characterized by the accumulation in mesangial areas of immune complexes containing polymeric IgAl. While epidemiology and clinical studies of IgA nephropathy are well established, the mechanism(s) underlying disease development is poorly understood. The pathogenesis of this disease involves the deposition of polymeric and undergalactosylated IgAl in the mesangium. Quantitative and structural changes of IgAl play a key role in the development of the disease due to functional abnormalities of two IgA receptors: The Fc $\alpha R$ (CD89) expressed by blood myeloid cells and the transferrin receptor (CD71) on mesangial cells. Abnormal IgA induce the release of soluble CD89 which is responsible for the formation of circulating IgA complexes. These complexes may be trapped by CD71 that is overexpressed on mesangial cells in IgA nephropathy patients allowing pathogenic IgA complex formation. $\diamond$
Meadow SR, Scott DG. Berger disease: HenochSchonlein syndrome without the rash. J Pediatr 1985; 106: 27-32.

6. Berger J, Yaneva H, Nabarra B, Barbanel C. Recurrence of mesangial deposition of IgA after renal transplantation. Kidney Int 1975; 7: 232-41.

7. Ponticelli C, Traversi L, Feliciani A, et al. Kidney transplantation in patients with IgA mesangial glomerulonephritis. Kidney lnt 2001; 60: 1948-54.
8. Berger $J$.

Glomérulonéphrites idiopathiques à dépôts mésangiaux d'IgA. In: Hamburger J, Crosnier J, Günfeld JP, eds. Néphrologie. Paris: Flammarion/MédicineSciences, 1979: 541-8.

9. Valentijn RM, Radl J, Haaijman JJ, et al. Circulating and mesangial secretory componentbinding IgA-l in primary IgA nephropathy. Kidney Int 1984; 26: 760-6.

10. Monteiro RC, HalbwachsMecarelli L, Roque-Barreira MC, et al. Charge and size of mesangial IgA in IgA nephropathy. Kidney Int 1985; 28: 666-71.

11. Rifai A, Small PA Jr, Teague PO, Ayoub EM. Experimental IgA nephropathy. J Exp Med 1979; 150: 1161-73.
12. van den Wall Bake AW, Bruijn JA, Accavitti MA, et al. Shared idiotypes in mesangial deposits in IgA nephropathy are not disease-specific. Kidney Int 1993; 44: 65-74.

13. de Fijter JW, Eijgenraam JW, Braam CA, et al. Deficient IgAl immune response to nasal cholera toxin subunit $B$ in primary $\lg A$ nephropathy. Kidney Int 1996; 50: 952-61.

14. Barratt J, Bailey EM, Buck KS, et al. Exaggerated systemic antibody response to mucosal Helicobacter pylori infection in IgA nephropathy. Am J Kidney Dis 1999; 33: 1049-57.

15. Layward L, Allen AC, Harper S), Hattersley JM, Feehally J. Increased and prolonged production of specific polymeric IgA after systemic immunization with tetanus toxoid in IgA nephropathy. Clin Exp Immunol 1992; 88: 394-8.

16. Feehally J. IgA nephropathy: a disorder of IgA production? Q J Med 1997; $90: 387-90$.

17. Allen AC, Bailey EM, Brenchley PE, et al. Mesangial lgAl in IgA nephropathy exhibits aberrant 0 -glycosylation: observations in three patients. Kidney Int 2001; 60: 969-73.

21. Hiki Y, Tanaka A, Kokubo T, et al. Analyses of IgAl hinge glycopeptides in IgA nephropathy by matrixassisted laser desorption/ionization time-of-flight mass spectrometry. J Am Soc Nephrol 1998; 9: 577-82.

22. Allen AC, Topham PS, Harper SJ, Feehally J. Leucocyte beta 1,3 galactosyltransferase activity in IgA nephropathy. Nephrol Dial Transplant 1997; 12: 701-6.

23. Kokubo T, Hiki Y, Iwase $H$, et al. Protective role of IgAl glycans against IgAl self-aggregation and adhesion to extracellular matrix proteins. J Am Soc Nephrol 1998; 9: 2048-54.

24. Tomana M, Novak J, Julian BA, Matousovic K, Mestecky J. Circulating immune complexes in IgA nephropathy consist of IgAl with galactosedeficient hinge region and antiglycan antibodies. J Clin Invest 1999; 104: 73-81.

25. Novak J, Julian BA, Tomana $M$, Mestecky J. Progress in molecular and genetic studies of IgA nephropathy. J Clin Immunol 2001; 21: 310-27.

26. Monteiro RC, van de Winkel JGJ. IgA Fc receptors. Annu Rev Immunol 2003; 21: 177-204.

18. Allen AC, Willis FR, Beattie TJ, Feehally J. Abnormal IgA glycosylation in HenochSchonlein purpura restricted to patients with clinical nephritis. Nephrol Dial Transplant 1998; 13: 930-4.

19. Sano T, Hiki Y, Kokubo T, et al. Enzymatically deglycosylated human IgAl molecules accumulate and induce inflammatory cell reaction in rat glomeruli. Nephrol Dial Transplant 2002; 17: 50-6.

20. Greer MR, Barratt J, Harper SJ, Allen AC, Feehally J. The nucleotide sequence of the IgAl hinge region in IgA nephropathy. Nephrol Dial Transplant 1998; 13: 1980-3.
27. Grossetete B, Launay $P$, Lehuen A, et al. Downregulation of Fc $\alpha$ receptors on blood cells of IgA nephropathy patients: evidence for a negative regulatory role of serum IgA. Kidney Int 1998; 53: 1321-35.

28. Launay P, Grossetete $B$, Arcos-Fajardo M, et al. Fca receptor (CD89) mediates the development of immunoglobulin A ( $\lg \mathrm{A})$ nephropathy (Berger's disease). Evidence for pathogenic soluble receptor-IgA complexes in patients and CD89 transgenic mice. $J$ Exp Med 2000; 191: 1999-2009. 
29. Muso $\varepsilon$, Yoshida $\varepsilon$, Takeuchi $\varepsilon$, et al. Enhanced production of glomerular extracellular matrix in a new mouse strain of high serum IgA ddy mice. Kidney Int 1996; 50: 1946-57.

30. Zheng F, Kundu GC, Zhang Z, et al. Uteroglobin is essential in preventing immunoglobulin A nephropathy in mice. Nat Med 1999; 5: 1018-25.

31. Silvain C, Patry C, Launay P, Lehuen A, Monteiro R. Altered expression of monocyte IgA Fc receptors is associated with defective endocytosis in patients with alcoholic cirrhosis. J Immunol 1995; 155: 1606-18.

32. Grossetête B, Viard JP, Lehuen A, Bach JF, Monteiro R. Impaired Fca receptor expression is linked to increased immunoglobulin A levels and disease progression in HIV-1infected patients. AIDS 1995; 9: 229-34.

33. Montenegro V, Chiamolera $M$, Launay P, Gonçalves CR, Monteiro RC. Impaired expression of IgA Fc receptors (CD89) by blood phagocytic cells in ankylosing spondylitis. J Rheumatol 2000; 27: 411-7.

34. Westerhuis R, Van Zandbergen $G$, Verhagen $\mathrm{NA}$, et al. Human mesangial cells in culture and in kidney sections fail to express Fc $\alpha$ receptor (CD89). J Am Soc Nephrol 1999; 10: 770-8.

35. Barratt J, Greer MR, Pawluczyk IZ, et al. Identification of a novel Fc $\alpha$ receptor expressed by human mesangial cells. Kidney Int 2000; 57: 1936-48.

36. Shibuya A, Sakamoto $N$, Shimizu $Y$, et al. Fc $\alpha / \mu$ receptor mediates endocytosis of IgM-coated microbes. Nat Immunol 2000; 1: 441-6.

37. McDonald KJ, Cameron AJ, Allen JM, Jardine AG. Expression of Fc $\alpha / \mu$ receptor by human mesangial cells: a candidate receptor for immune complex deposition in IgA nephropathy. Biochem Biophys Res Commun 2002; 290: 438-42.
38. Moura IC, Centelles MN, Arcos-Fajardo $M$, et al. Identification of the transferrin receptor as a novel immunoglobulin (Ig)Al receptor and its enhanced expression on mesangial cells in IgA nephropathy. J Exp Med 2001 ; 194: 417-25.

39. Haddad $\varepsilon$, Moura IC, ArcosFajardo $M$, et al. Enhanced expression of the CD71 mesangial IgAl receptor in Berger's disease and Henoch-Schönlein nephritis: association between CD71 expression and IgA deposits. J Am Soc Nephrol 2003; 14: 327-37.

40. Chen A, Chen WP, Sheu LF, Lin Cy. Pathogenesis of IgA nephropathy: in vitro activation of human mesangial cells by IgA immune complex leads to cytokine secretion. J Pathol 1994; 173: 119-26.

41. Van den Dobbelsteen ME, van der Woude FJ, Schroeijers WE, et al. Binding of dimeric and polymeric IgA to rat renal mesangial cells enhances the release of interleukin 6 . Kidney Int 1994; 46: 512-9.
42. Duque N, Gomez-Guerrero C, Egido J. Interaction of IgA with Fc $\alpha$ receptors of human mesangial cells activates transcription factor nuclear factor $-\kappa B$ and induces expression and synthesis of monocyte chemoattractant protein$1, \mathrm{IL}-8$, and IFN-inducible protein 10. J Immunol 1997; 159: 3474-82.

43. Schena F, Cerullo G, Rossini $M$, et al. Increased risk of end-stage renal disease in familial IgA nephropathy. J Am Soc Nephrol 2002; 13: 453-60.

44. Hsu S, Ramirez S, Winn M, Bonventre J, Owen W. Evidence for genetic factors in the development and progression of IgA nephropathy. Kidney Int 2000; 57: 1818-35.

45. Ghavari A, Scolari F, Schena $F$, et al. IgA nephropathy, the most common cause of glomerulonephritis, is linked to 6q22-23. Nat Genet 2000 ; 26 : 354-7.

\section{TIRÉS À PART}

R.C. Monteiro 